\title{
Self-Assembled Monolayers on C(0001)
}

\author{
Fernando Terán Arce, ${ }^{*}$ José L. Zubimendi, Maria E. Vela, \\ Roberto C. Salvarezza, and Alejandro J. Arvia
} Instituto de Investigaciones Fisicoquímicas Teóricas y Aplicadas. (INIFTA), Universidad Nacional de La Plata-Consejo Nacional de Investigaciones Científicas y Técnicas, La Plata, Argentina

*Present address: Center for Nanomedicine, Department of Medicine, University of Chicago, Chicago, IL, USA

\section{Contents}

20.1 Introduction

20.2 Characteristic of the HOPG Substrate

\subsection{INTRODUCTION}

Scanning nanoscopies have led to a new stage in the study of interfacial processes. Data derived from these techniques, especially scanning tunneling microscopy (STM) and atomic force microscopy (AFM), offer the possibility of studying the physical chemistry of surfaces on solid substrates at the atomic and molecular level [1-5].

Heterogeneous catalysis is an important field for the application of these techniques. Because of the use of these nanoscopies, advances have been made in the knowledge of the geometry and effective area of solid catalysts, the sintering process that decreases their performance and lifetime, the adsorbate film structure on crystallographically well-defined surfaces, and the influence of surface defects on the dynamic behavior of these films during adsorption, desorption, and chemical reaction stages. 
Nanometer-scale $(\mathrm{nm})$ nanoscopies provide information on restricted molecular domains that comprise some hundreds of molecules. This information at the local level is not accessible by other surface analysis techniques because the latter provide average data on the whole sample. Studies at the local level reveal the complexity of physicochemical processes taking place at solid/fluid interfaces under different perturbation conditions. Local data are a solid basis for the theoretical interpretation of these processes by the use of Quantum Mechanics procedures.

Nanoscopies supplemented with conventional techniques will allow the rational handling of the catalyst/reactive system based on its knowledge at the atomic/molecular level. The application of nanoscopies in surface chemistry offers the possibility for determining the nanostructure of solid surfaces, surface reconstruction phenomena, to identify the structure of ionic and molecular adlayers, to study the dynamics of these adlayers in their adsorption and desorption at the submonolayer and monolayer (ML) level. Likewise, they are important tools to follow reactions at solid surfaces in real time in different environments. The reader can get acquainted with the state of the art on these topics in Refs [5-12].

This chapter describes the application of tunneling and AFM to the study of inorganic and organic adsorbates on $\mathrm{C}(0001)$ at the submonolayer and ML level. The $\mathrm{C}(0001)$ surface can be taken as a model system for the study of adsorption processes because it is atomically smooth and exhibits a low chemical reactivity, allowing an easy handling in the atmosphere. The knowledge of adsorption on carbon is important in the field of electrocatalysis because carbon is widely used as a matrix for the dispersion of catalytically active metallic clusters.

\subsection{Characteristic of the hOPG Substrate}

\subsubsection{General Considerations}

Highly oriented pyrolytic graphite (HOPG) is the most adequate type of carbon to investigate the adsorption of both molecules and atoms, and the formation of molecular and atom clusters on the $\mathrm{C}(0001)$, the basal plane of graphite [1-5],[9-12] The procedure for HOPG fabrication was developed by Union Carbide in USA. HOPG is prepared from the thermal decomposition of gaseous hydrocarbons on a surface heated at $1200-3800^{\circ} \mathrm{C}$ followed by highpressure compression of the surface under heating [13].

HOPG's first use was as an X-ray diffraction grating. Later, with the advent of nanoscopies it became of particular interest as a carbon material with flat terraces constituted by the basal plane that could be resolved at the atomic scale. Accordingly, HOPG was utilized as a calibration standard for STM, and as a substrate for adsorption studies. Besides, the ease with which HOPG can have a 
pristine basal plane surface just by exfoliation with scotch tape and its chemical inertia make HOPG a very important substrate to be utilized in STM and AFM.

HOPG consists of ordered layers (graphene sheets) of carbon atoms constituting a honeycomb lattice. The arrangement of graphene sheets is of the type A.B.A.B (Fig. 20.1(a)), the nearest neighbor graphene sheets are shifted horizontally by one interatomic distance [14]. The separation distance of nearest neighbor graphene sheets is $0.355 \mathrm{~nm}$, and the lattice constant in the vertical direction is $0.67 \mathrm{~nm}$. Correspondingly, for alternatively located graphene sheets, three carbon atoms out of the six atoms forming each hexagon of the 2D lattice lie on the same vertical, whereas the remaining three carbon atoms lie on the vertical containing the center of hexagons (Fig. 20.1(b)).

For each graphene sheet the atomic lattice consists of six carbon atoms forming an open honeycomb type hexagon with $0.142 \mathrm{~nm}$ between nearest neighbor atoms. The Bravais lattice, however, corresponds to a hexagonal lattice centered with two carbon atoms for each unit cell, and $0.246 \mathrm{~nm}$ separation between neighbor Bravais point so that $0.246 / 0.142=\sqrt{3}$. The interaction between graphene layers is determined by van der Waals weak forces, making exfoliation of HOPG easy.

Graphite is thermodynamically stable under usual conditions, but its structure is typically anisotropic as it is reflected, for instance, by the Young's modulus that is $10.3 \times 10^{-5} \mathrm{MPa}$ along the basal plane and $0.3 \times 10^{-5} \mathrm{MPa}$ in the direction perpendicular to the basal plane. A similar effect occurs with the capacitance of the HOPG/aqueous electrolyte interface, the potential of zero charge, and the work function values (see Chapter 21).

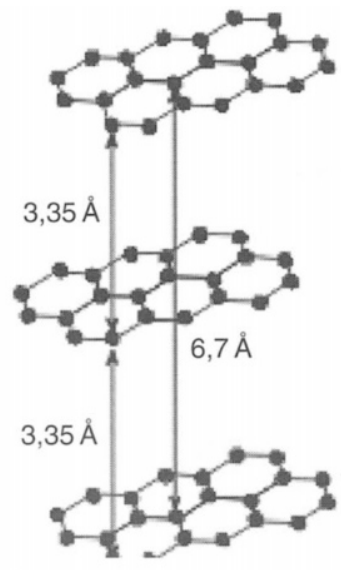

(a)

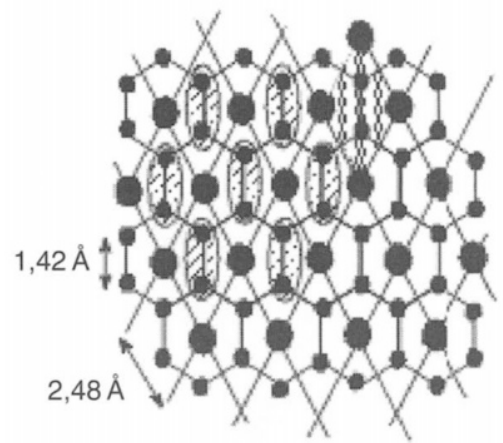

(b)

Figure 20.1 (a) Scheme of the 3D highly oriented pyrolytic graphite (HOPG) atomic layer. Note the lateral displacement of atomic layers. (b) Little circles form the honeycomb lattice. Big circles correspond to the Bravais cell. The unit cell is drawn (shadow) at the upper right part of the figure. The corrugation between two carbon atoms located within the ellipse is the region sensed by the tip in the contact mode atomic force microscopy (AFM). 
The four valence electrons of carbon are involved in three $\sigma$ bonds and one $\pi$ bond with its neighbors in plane. The electrical conductivity of graphite is due to $\pi$ bonded electrons. In contrast to insulator diamond, the electrical resistance of graphite along the basal plane direction is $4.1 \times 10^{-5} \Omega \mathrm{cm}$, a figure of the same order of magnitude as that of metals such as platinum and palladium. According to the band theory, graphite is considered as a semimetal, the overlapping of the conduction and valence bands is about $0.04 \mathrm{eV}$. The electronic structure of graphite accounts for its hydrophobicity [15].

\subsubsection{Nanoscopy Characterization of HOPG}

\subsubsection{The hexagonal lattice}

AFM images $\left(10 \times 10 \mu \mathrm{m}^{2}\right)$ of a fresh HOPG surface (Fig. 20.2) show a number of features, namely, large monoatomic terraces about $100 \mathrm{~nm}$ wide and several micrometer long. Terraces are separated by steps of either one atom or a few atoms in height. Some triangular-shaped terraces with angles that are multiples of $30^{\circ}$ are consistent with the hexagonal lattice (Fig. 20.3(a)). Besides, STM images also show some features that are artifacts from the exfoliation technique (Fig. 20.3(b)). These artifacts have been classified as steps, strings, fibers [16] either single or agglomerated, small pieces of graphite, and very tiny particles. A detailed analysis of these additional features is required to avoid a wrong interpretation of the structure of adsorbate patterns on HOPG.

When terraces are imaged at high resolution (Fig. 20.4(a)), i.e., below $10 \times 10 \mathrm{~nm}^{2}$, the STM image of C(0001) depicts a hexagonal lattice with nearest neighbor distance $d=0.246 \mathrm{~nm}$. The corrugation of this type of image depends

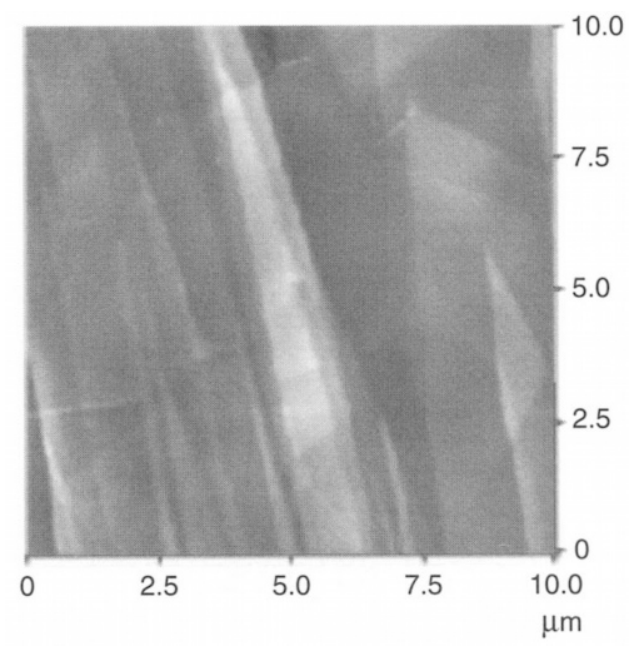

Figure 20.2 Ex situ atomic force microscopy (AFM) image of the basal plane of highly oriented pyrolytic graphite (HOPG). Wide terraces separated by steps can be seen. 


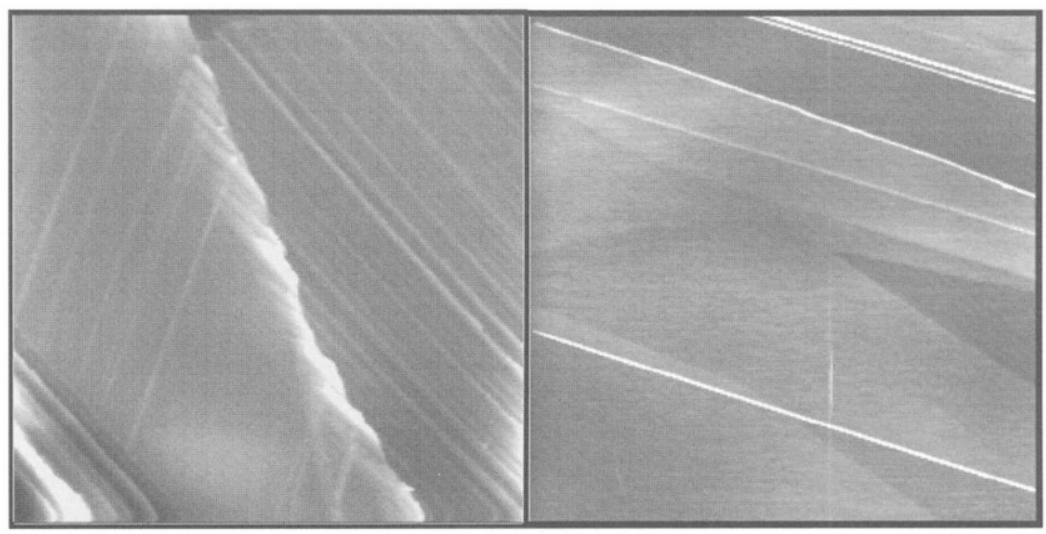

(a)

(b)

Figure 20.3 (a) Ex situ $3.8 \times 3.8 \mu \mathrm{m}^{2}$ atomic force microscopy (AFM) image of highly oriented pyrolytic graphite (HOPG) that shows steps of different heights. (b) A $4.15 \times 4.15 \mu \mathrm{m}^{2}$ AFM image of HOPG where strings produced by the exfoliation technique are shown.

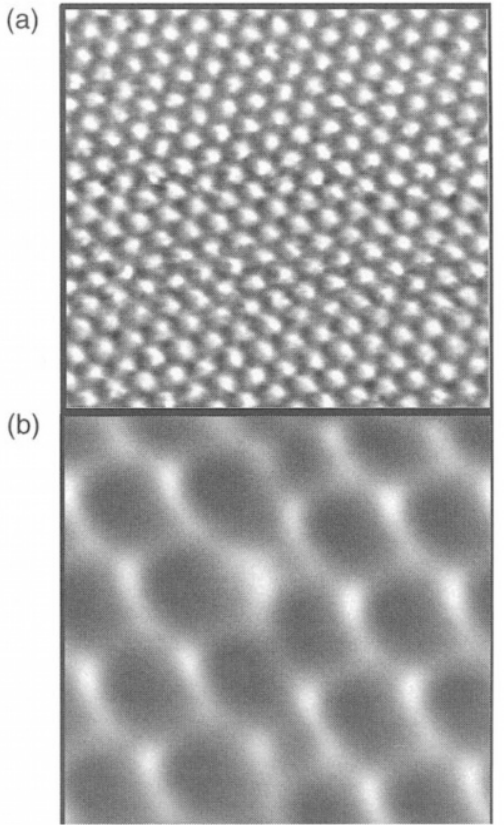

Figure 20.4 (a) Ex situ $3 \times 3 \mathrm{~nm}^{2}$ scanning tunneling microscopy (STM) image usually found in highly oriented pyrolytic graphite (HOPG). (b) Honeycomb structure observed by STM $\left(1 \times 1 \mathrm{~nm}^{2}\right)$. 
on the tunneling current $\left(I_{t}\right)$ and the voltage $\left(V_{t}\right)$ applied between the STM tip and the sample surface. Thus, corrugation of about $0.1 \mathrm{~nm}$ results for $I_{\mathrm{t}} \approx 1 \mathrm{nA}$ and $V_{\mathrm{t}} \approx 0.05 \mathrm{~V}$, whereas the corrugation decreases to $0.02 \mathrm{~nm}$ for $V_{\mathrm{t}} \approx 1 \mathrm{~V}$. The change in voltage polarity has practically no effect on the HOPG image. Occasionally, the typical honeycomb structure of graphite can be observed by STM (Fig. 20.4(b)). As discussed below, the origin of this type of images is controversial. They have been considered as "high-resolution images" that are obtained when the STM tip is extremely sharp or as an "artifact" arising from a multiple tip [17].

The lattice shown in Fig. 20.1(b), which is usually imaged by STM or AFM, is formed by only three instead of six carbon atoms. The corresponding nearest neighbor carbon-carbon atom distance is that of the Bravais hexagonal lattice referred to above. The scheme depicted in Fig. 20.1(a, b) accounts for the appearance of this image.

\subsubsection{Additional features}

Step corrugations of about $10-20 \mathrm{~nm}$ are easily observed with thin graphite samples that have been exfoliated several times. However, the Bravais lattice can be observed by small-size imaging $\left(\approx 10 \times 10 \mathrm{~nm}^{2}\right)$, and at slightly higher magnifications $\left(50 \times 50 \mathrm{~nm}^{2}\right)$ superstructures of different periodicity are occasionally observed. These superstructures of HOPG (Fig. 20.5.) make it difficult to recognize unambiguously the structure of molecular adsorbates.

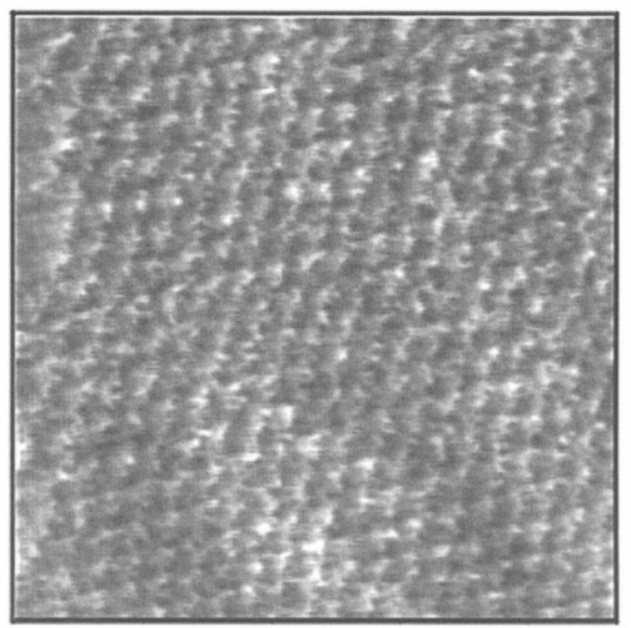

Figure 20.5 Ex situ $46 \times 46 \mathrm{~nm}^{2}$ atomic force microscopy (AFM) image of highly oriented pyrolytic graphite (HOPG) showing a lattice that is not correspondent with the lattice shown in Fig. 20.1. 
Strings are thin graphite stripes that are removed by exfoliation from steps and attached to another step [16]. The three threads shown in Fig. 20.3(b) that covered the entire image are $66 \mathrm{~nm}$ large and about $2.8 \mathrm{~nm}$ wide.

Fibers are observed by STM as thin tubes about $2.5 \mathrm{~nm}$ in diameter and $20 \mathrm{~nm}$ long formed by agglomeration of threads. Atomic resolution at fibers can also be obtained, although with a poor definition. They are produced by step rupture by exfoliation.

Island-like pieces, most of them at the border of holes, are also sometimes produced by exfoliation (Fig. 20.6). The islands depicted in this figure are about $0.3 \mathrm{~nm}$ high, a figure that is similar to the depth of holes.

Different superlattices with $\sqrt{3} \times \sqrt{3}$ periodicity have been imaged. This periodicity has been related to rotation of graphite lattice [17]. These superlattices can be produced by either a multiple tip effect $[17 \mathrm{~b}]$ or electronic perturbations caused by adsorbed molecules [17c]. A hexagonal superlattice with a $4.4 \mathrm{~nm}$ periodicity, rotated $30^{\circ}$ with respect to the HOPG lattice, and $0.38 \mathrm{~nm}$ corrugation has also been reported [17a]. This superlattice was also attributed to rotation of the surface layer of graphite. As this type of superstructures is most frequently observed for thin layers of material, they have been associated with charge density waves $[14,18]$.

Occasionally, a sort of lattice of holes is also imaged. The structure of this lattice can be interpreted as an atomic honeycomb lattice in which each hole in the image would represent the hole of a hexagon in the honeycomb lattice. According to theoretical calculations, graphite STM images with atomic

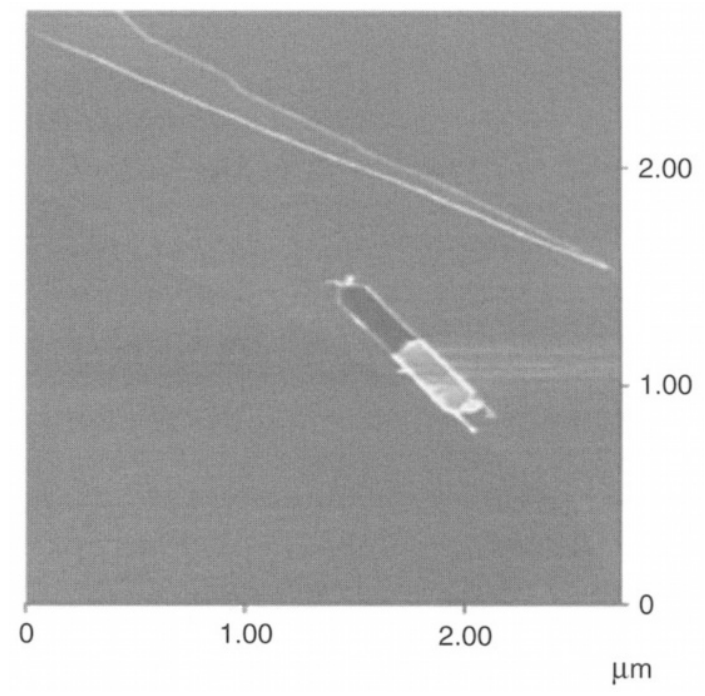

Figure 20.6 Ex situ atomic force microscopy (AFM) image that shows island-like pieces produced on highly oriented pyrolytic graphite (HOPG) by step rupture from the exfoliation procedure. 
resolution should be dominated by independent Fourier components [19] of three carbon atoms usually imaged. The multiple tip effect would produce a relative change in the amplitude and phase of other components, this fact being reflected in the change of the maximum amplitude observed by STM. A poor instrument resolution might produce a comparable effect [20]. The same features from atomic resolution AFM images of graphite (Fig. 20.7(a, b)) can be distinguished.

Two models have been proposed to explain the AFM images of graphite [21]. In one of these models the calculations are based on the scanning of the graphite surface with a single potassium atom. For applied forces of the order of $1 \mathrm{nN}$, i.e., a value lower than about $50 \mathrm{nN}$ used in the contact mode AFM, the corrugation between two carbon atoms located within the ellipse (Fig. 20.1(b)) would be indistinguishable by the AFM cantilever tip. But the situation would be reversed when the tip goes through two ellipses via the hexagon centers.

Another possibility considers the asymmetry of carbon sites in the graphite lattice (Fig. 20.1(a)). Thus, the carbon atom located in the upper graphene, which is directly above the carbon atom in the lower graphene, would suffer a weaker interaction with the tip than that facing the centers of the hexagon. This explanation would be similar to that admitted for the interpretation of the corresponding STM images.

Therefore, it can be concluded that STM imaging on HOPG is influenced by structural defects, adsorbates and electronic effects [22]. The latter would prevail at step sites where an asymmetric distribution of electric charges would be more favorable.

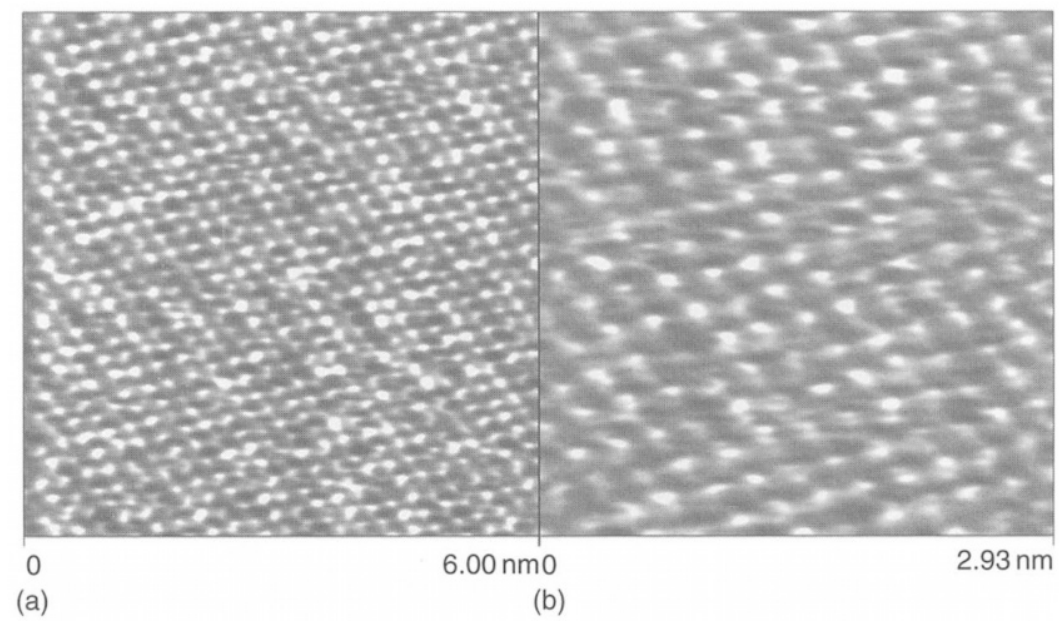

Figure 20.7 Ex situ atomic force microscopy (AFM) images of highly oriented pyrolytic graphite (HOPG). The distance is $0.246 \mathrm{~nm}$. 


\subsection{Self-Assembled Submonolayers and MONOLAYERS}

One important aspect of heterogeneous chemical reactions at solid surfaces is related to the presence of adsorbed species that play a key role in determining the rate and efficiency of these processes. Therefore, the knowledge of molecular arrangements on solid catalysts of reactants, reaction intermediates, or products is of outstanding importance in dealing with fundamental aspects of heterogeneous catalysis.

Self-assembled molecular arrangements on HOPG can be spontaneously produced by different procedures that are based on the type of interactions between either bare HOPG regions or functional oxygen-containing groups existing at the HOPG surface (see Chapter 21). These arrangements, covering from the submonolayer to the multilayer level, are dominated by either physical or chemical adsorption.

The adsorption of an atom on a molecule at a solid surface is attributed to a physisorption phenomenon principally because of van der Waals forces. In physisorption no appreciable reordering in the adsorbate electronic distribution occurs. This situation is generally found in the adsorption of noble gases on metal surfaces, in which adsorption energy values in the range $1-10 \mathrm{~kJ} / \mathrm{mol}$ are involved. These figures are of the same order of magnitude as that of the thermal energy $(k T)$ of molecules at $T=298 \mathrm{~K}$ (approximately $2.5 \mathrm{~kJ} / \mathrm{mol}$ ). Accordingly, to observe physisorbed systems by atomic resolution STM or AFM, experiments have to be performed at a very low temperature and above 1 atm pressure [23].

The formation of supramolecular layers (see Chapter 21) is another way of producing adequate architectural molecular designs on HOPG and carbons in general, although the structural analysis of these layers by nanoscopic techniques is still a complicated matter. A few typical examples of adsorbates on $\mathrm{C}(0001)$ are described in the following sections.

\subsubsection{Alkane Adsorption on C(0001)}

In principle, the adsorption of alkane molecules on $\mathrm{C}(0001)$ would appear unlikely because of the inert character of the substrate. In this case, however, besides van der Waals forces, other contributions come into play and can make energy adsorption reach values of up to $100 \mathrm{~kJ} / \mathrm{mol}$, which are comparable to those of chemisorption processes. This enables determining the structure of aliphatic hydrocarbons adsorbed on $\mathrm{C}(0001)$ by AFM or STM because the adsorbate withstands tip-sample interaction forces.

The adsorption energy of alkanes on $\mathrm{C}(0001)$ decreases with temperature and increases with the chain length [24] due to an increase in the affinity of alkane carbon atoms with $\mathrm{C}(0001)$ atoms. This involves the adsorption of aliphatic molecules ordered with the chain axis lying parallel to the $C(0001)$ plane. Under these conditions, the interaction of the adsorbed molecule increases because of 
the geometric matching of the carbon lattice of the $\mathrm{C}(0001)$ plane with that of the zigzag aliphatic chain, each $\mathrm{CH}_{2}$ occupying the hexagon area in the graphite lattice. With this configuration the adsorption energies are $21.6 \mathrm{~kJ} / \mathrm{mol}$ for $n$-hexane and $105.6 \mathrm{~kJ} / \mathrm{mol}$ for $n$-hexadecane.

\subsubsection{Sulfur Atom Submonolayers on HOPG}

Sulfur electroadsorbs on HOPG from $\mathrm{SH}^{-}$-containing neutral buffered aqueous solution $(\mathrm{pH} 8)$ at potentials $(E)$ close to $-0.8 \mathrm{~V}$ (versus NHE), i.e., at values of $E$ more negative than the reversible potential $\left(E_{\mathrm{r}}\right)$ for the $\mathrm{SH}^{-}=\mathrm{S}+\mathrm{H}^{+}+\mathrm{e}^{-}$ reaction. The surface coverage by sulfur atoms, estimated from the electroadsorption/electrodesorption charge, is close to $1 / 2$. Different structures of sulfur atom submonolayers on HOPG have been observed by STM [25a, b]. One of these structures corresponds to sulfur trimers with $d=0.24 \mathrm{~nm}$ and $\mathrm{S}$ atoms atop C atoms (Fig. 20.8(a)). Conversely, for $E>E_{\mathrm{r}}$, other submonolayer structures are formed, namely, a $\sqrt{3} \sqrt{ } 3 \mathrm{R} 30^{\circ}$ structure with $d=0.42 \mathrm{~nm}$, a sulfur atom honeycomb lattice with $d=0.24 \mathrm{~nm}$, rectangular arrays of sulfur atoms with $d=$ $0.21 \mathrm{~nm}$ (Fig. 20.8(b)). The influence of the HOPG surface on sulfur atom electroadsorption is reflected in the values of $d=0.42 \mathrm{~nm}$ and $d=0.24 \mathrm{~nm}$, whereas the S-S distance, $d=0.21 \mathrm{~nm}$, which is observed for $E>E_{\mathrm{r}}$, is close to that found for polysulfide species [26]. A similar behavior has been observed for sulfur atom electroadsorption on $\mathrm{Au}(111)$ surfaces [26]. Adsorption energy values for $\mathrm{S}$ atom adsorption on HOPG in the range $30-40 \mathrm{~kJ} / \mathrm{mol}$ have been evaluated theoretically $[25 b]$.

\subsubsection{Alkanethiol Adsorption on C(0001)}

The contrast of organic molecules adsorbed on C(0001) in STM images depends on the functional group at the molecule head [27]. Contrast is generally enhanced for functional groups than for aliphatic chains. For functional groups it decreases in the order $\mathrm{SH}>\mathrm{I}>\mathrm{Br}>\mathrm{NH}_{2}$. This sequence offers the possibility to discriminate the functional group from the rest of the molecule by STM. It should be noted that for $\mathrm{OH}$ and chloride groups, contrast is comparable to that of the remaining aliphatic chain, which turns their distinction by STM practically impossible.

The structures of the $\mathrm{CH}_{3}\left(\mathrm{CH}_{2}\right)_{22} \mathrm{SH}$ adlayers on $\mathrm{C}(0001)$ [28] are similar to those of the alkanes. They consist of molecular domains lying parallel to each other forming a $90^{\circ}$ angle with respect to the chain direction. A kind of disorder is also observed in the vicinity of neighbor SH groups.

Ex situ AFM images of a 1-dodecanethiol ML on C(0001) (Fig. 20.9(a)) exhibit an array of parallel-oriented bright rows [29]. At a higher resolution (Fig. 20.9(b)) pale bands between rows, corresponding to aliphatic chains, and bright circles along each row, attributed to $\mathrm{S}$ heads, can be seen. Similar images 


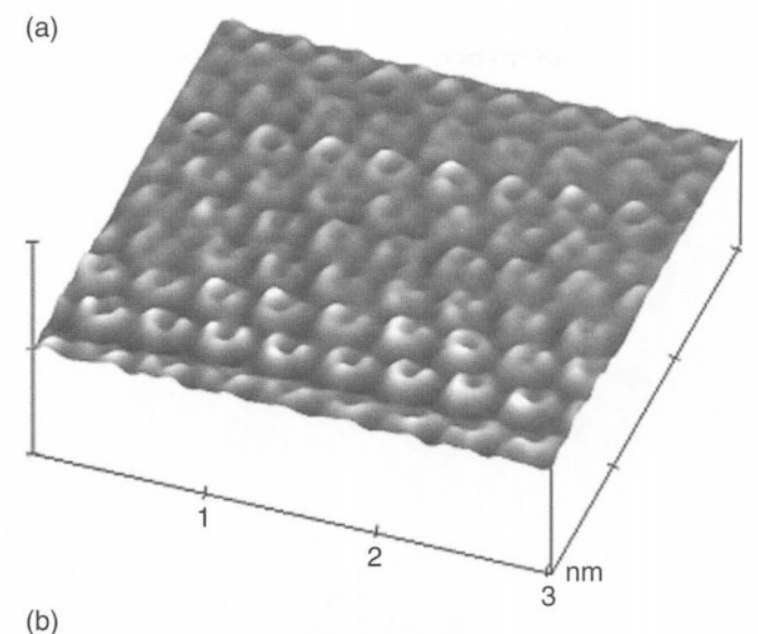

(b)

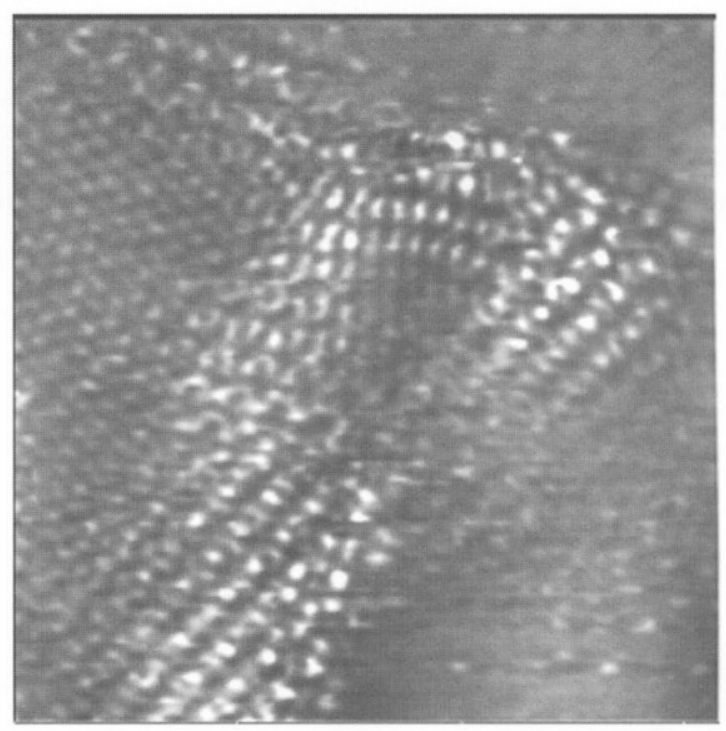

Figure 20.8 Ex situ atomic resolution scanning tunneling microscopy (STM) images of sulfur atoms adsorbed on highly oriented pyrolytic graphite (HOPG): (a) $3 \times 3 \mathrm{~nm}^{2}$; (b) $6.32 \times 6.32 \mathrm{~nm}^{2}$.

are obtained by STM (Fig. 20.10) although, in this case, pale bands cannot be seen. Bands $1.2 \mathrm{~nm}$ in length become somewhat shorter than that of the extended chain molecule. The interband separation, which would be related to the intermolecular separation, is $0.65 \mathrm{~nm}$. The angle between a row of $S$ heads and the chain direction is $120^{\circ}$. The $\mathrm{S}$ heads along a row are generally placed behind the chain of the neighbor row. 


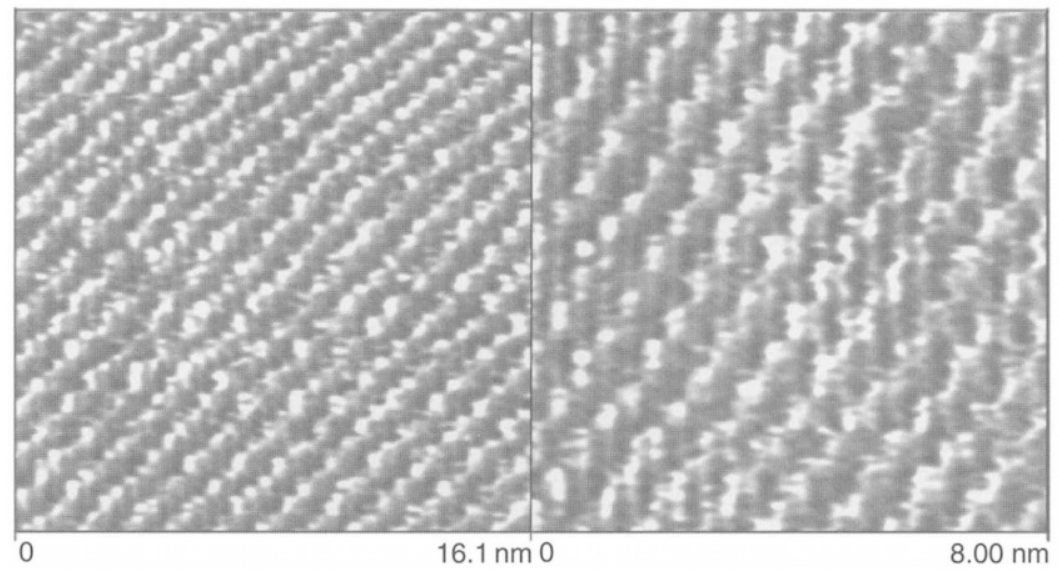

(a)

(b)

Figure 20.9 Atomic force microscopy (AFM) images of 1-dodecanethiol monolayer adsorbed on C(0001). (a) Bright spots are attributable to sulfur heads. Image (a) exhibits an array of parallel-oriented bright rows. At a higher resolution (b) pale bands between rows corresponding to aliphatic chains and bright circles along each row attributed to sulfur heads can be seen.

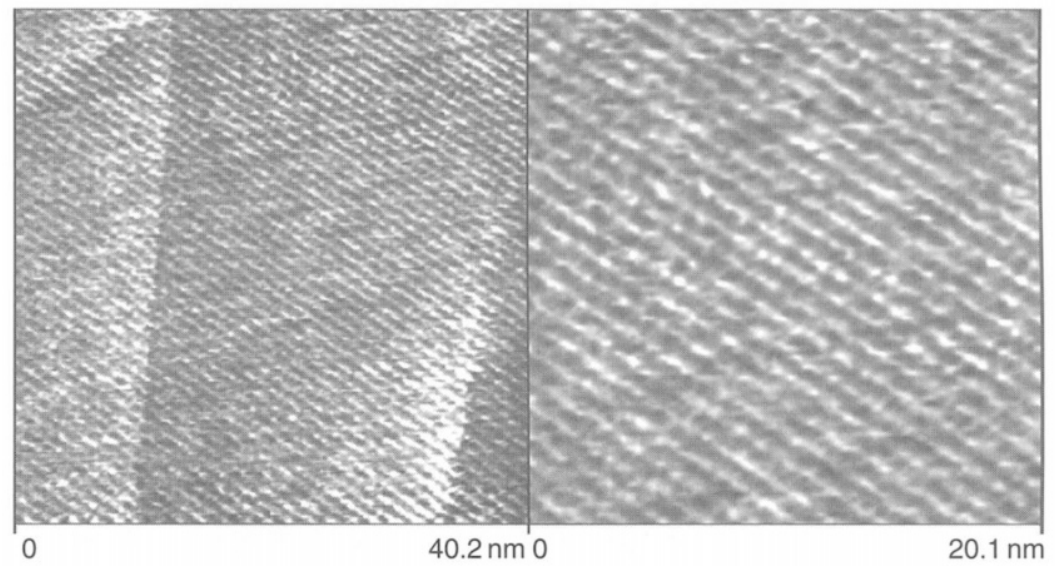

(a)

(b)

Figure 20.10 Scanning tunneling microscopy (STM) images of 1-dodecanethiol monolayer adsorbed on C(0001). (a) and (b) Sulfur heads exhibit an array of parallel-oriented bright rows, At a higher resolution only the highly oriented pyrolytic graphite (HOPG) lattice can be observed (not shown). 


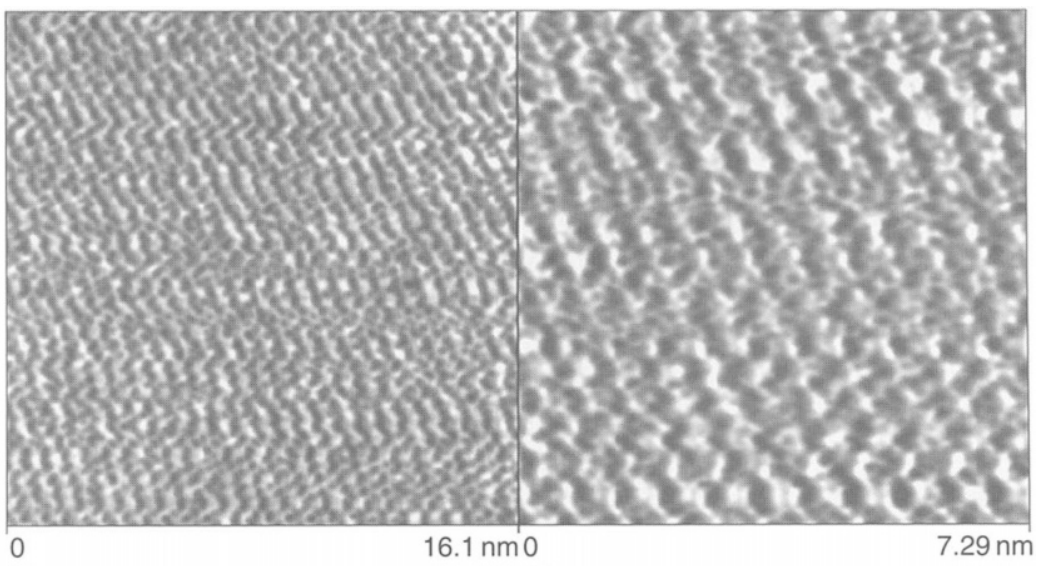

(a)

(b)

Figure 20.11 Molecular resolution atomic force microscopy (AFM) images of a 1-butanethiol monolayer on highly oriented pyrolytic graphite (HOPG). Distance between bright lines are compatible with the length of adsorbed molecules.

Ex situ AFM images of 1-butanethiol on C(0001) (Fig. 20.11) exhibit a structure similar to that described above. In the lower left part of the image depicted in Fig. 20.11 some bright spots along the rows, probably related to $\mathrm{S}$ heads, and a few pale bands, associated with aliphatic chains, can also be observed. The intermolecular separation distance between two bright spots is $0.45 \mathrm{~nm}$. The $0.55-\mathrm{nm}$-long band is consistent with the aliphatic chain length (Fig. 20.11). As observed for 1-dodecanethiol, the angle between the chains and the direction of a bright row of S heads is $115^{\circ}$.

Alkanethiols with short- and medium-length aliphatic chains adsorbed on $\mathrm{C}(0001)$ display heads with the molecular axis lying parallel to the basal plane of the substrate. This conclusion that was drawn from the analysis of AFM images is consistent with the interrow separation of S heads deduced from STM images, and agrees with previous results for alkanethiol with 22 carbon atoms [27, 28]. However, aliphatic chains of intermediate length seem to be extended on the substrate surface only a fraction of their length. As in the case of $\mathrm{Au}(111)$, for alkanethiols adsorbed on $\mathrm{C}(0001)$ the longer the adsorbate aliphatic chains the more ordered they are [30].

The fact that in some regions in the images (Figs 20.9 and 20.10) the length of 1-dodecanethiol does not match exactly that of the molecule fully extended on the surface is attributed to the occurrence of a mixed cis-trans configuration (gauche conformation). The scheme of this configuration (Fig. 20.12) includes $\mathrm{C}$ atoms located at the same sites as those of the $\mathrm{C}(0001)$ lattice, and the change in configuration is shown by an ellipse (see Fig. 20.12). The $S$ atom separation between two aliphatic chains in a row is $1.25 \mathrm{~nm}$, a figure that agrees with that determined from the images. The $\mathrm{S}$ atom separation between two molecules 


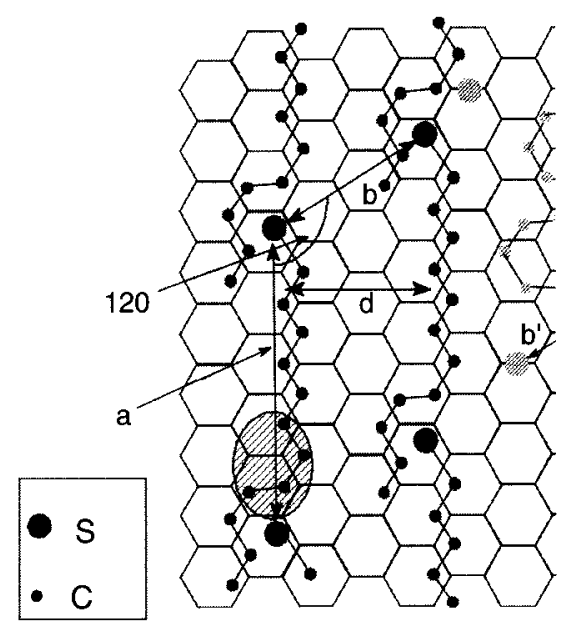

Figure 20.12 Scheme of the 1-dodecanethiol structure adsorbed on highly oriented pyrolytic graphite (HOPG). The axis of the hydrocarbon chain is oriented parallel to the surface, although partially extended.

located at neighbor rows is $0.75 \mathrm{~nm}$, and the angle formed between the molecule axis and the direction of $\mathrm{S}$ heads is $120^{\circ}$. These figures agree reasonably well with measured values.

The adsorption energy of alkanethiols on $\mathrm{C}(0001)$ can be estimated considering the energy of lateral interactions between the aliphatic chains, which is of the order of $4 \mathrm{~kJ} / \mathrm{mol}$ for $\mathrm{CH}_{2}$, and the intermolecular interaction energy in liquid alkanes, which for hexadecane is $57 \mathrm{~kJ} / \mathrm{mol}$. The energy difference for the interaction between the chains of the hexadecanethiol $\mathrm{ML}$ on $\mathrm{Au}(111)$ and in liquid alkane is $17 \mathrm{~kJ} / \mathrm{mol}$. From the calculation of the adsorption energy of $S$ on $C(0001)$ [31], it was concluded that the vertices of graphite hexagons (small circles in Fig. 20.1) or sites located between two hexagons (large circles in Fig. 20.1) are the most favorable adsorption sites, as shown by 1-butanethiol adsorption on $\mathrm{C}(0001)$. Predictions, however, become more uncertain because in these cases the tendency of $\mathrm{C}$ atoms to follow the $\mathrm{C}(0001)$ lattice prevails. On the basis of the adsorption energies of $n$-hexadecane $(100 \mathrm{~kJ} / \mathrm{mol}), n$-hexane $(22 \mathrm{~kJ} / \mathrm{mol})$, and $\mathrm{S}$ on $\mathrm{C}(0001)$, the adsorption energy for dodecanethiol obtained from extrapolation is $70 \mathrm{~kJ} / \mathrm{mol}$. This value exceeds that of the adsorption energy of $\mathrm{S}$ on $\mathrm{C}(0001)$ and confirms the stability of the adsorbate on $\mathrm{C}(0001)$.

The ordering of adlayers of alkanethiols on $\mathrm{C}(0001)$ indicates that the head-neighbor molecule chain interactions and, to a lesser extent, that of the head-head of molecular pairs prevail.

Results from the adsorption of alkanethiols on $\mathrm{C}(0001)$ as well as on $\mathrm{Au}(111)$ [32] show a strong influence of the substrate on the configuration of adsorbed molecules, regardless of the length of the aliphatic chain. 


\section{ACKNOWLEDGMENTS}

The authors thank the Consejo Nacional de Investigaciones Científicas y Técnicas (CONICET) and Agencia Nacional de Promoción Científica y Tecnológica from Argentina for their financial support (PIP 0897, PICT 99-5030, and PICT $98 \mathrm{~N}^{\circ} 06-03251$ ) to the research projects on which this chapter is based. MEV is a member of the research career of CIC.

\section{REFERENCES}

1. Binnig, G. and Rohrer, H. (1982). Surface studies by scanning tunneling microscopy. Helv. Phys. Acta, 55, 726.

2. Röhrer, H. (1989). Scanning tunneling microscopy and related methods. NATO ASI Series, Series E, Applied Sciences, No. 184, Kluwer, p. 1.

3. Forbes, R.G. (1989). Scanning tunneling microscopy and related methods. NATO ASI E Series, No. 184, Kluwer, p. 163.

4. Tersoff, J. and Hamann, D.R. (1983). Theory and application for scanning tunneling microscope. Phys. Rev. Lett., 50; Tersoff J. (1989). Scanning tunneling microscopy and related methods. NATO ASI E Series, No. 184, Kluwer, p. 77.

5. Leavens, C.R. and Aers, G.C. (1989). Scanning tunneling microscopy and related methods. NATO ASI E Series, No. 184, Kluwer, p. 27.

6. Siegenthaler, H. (1992). Scanning Tunnelling Microscopy II (R. Wiesendangerand and H.J. Guntherodt, eds). Springer-Verlag.

7. Gewirth, A.A. and Siegenthaler, H. (1995). Nanoscale probes of the solid/liquid interface. NATO ASI Series, Applied Sciences, No. 288, Kluwer.

8. Lorenz, H.J. and Plieth, W. (1998). Electrochemical nanotechnology. In situ Local Probe Techniques at Electrochemical Interfaces. Wiley-VCH.

9. Itaya, K. (1998). In situ scanning tunneling microscopy in electrolyte solutions. Progr. Surf. Sci., 58, 121-47.

10. Capella, B. and Dietler, G. (1999). Force-distance curves by atomic force microscopy. Surf. Sci. Rep., 34, 1-3.

11. Binns, G., Baker, S.H., Demangeat, C., and Parlebas, J.C. (1999). Growth, electronic, magnetic and spectroscopic properties of transition metals on graphite. Surf. Sci. Rep., 34, 107-70.

12. Binnig, G., Quate, C.F., and Gerber, C. (1986). Atomic force microscope.Phys. Rev. Lett.,56, 930-3.

13. Mc.Creery, R.L. (1994). Carbon electrodes: structural effects on electron transfer kinetics, electroanalytical chemistry, Vol. 17 (A.J. Bard, ed.). Marcel Decker, p. 221.

14. Wiesendanger, R. and Anselmetti, D. (1994). Scanning Tunneling Microscopy I. (H.J. Güntherodt and R. Wiesendanger, eds). Springer-Verlag.

15. Adamson, A.W. (1990). Physical Chemistry of Surfaces. Wiley-Interscience.

16. Chang, H. and Bard, A.J. (1991). Observation and characterization by scanning tunneling microscopy of structures generated by cleaving highly oriented pyrolytic graphite. Langmuir, 7, 1143-53. 
17. (a) Liu, C.-Y., Chang, H., and Bard, A.J. (1991). A large scale hexagonal domainlike structures superimposed on the atomic corrugation of a graphite surface observed by scanning tunneling microscopy. Langmuir, 7, 1138-42; (b) Albrecht, T.R., Mizes, H.A., Nogami, J., et al. (1988). Observation of tilt boundaries in graphite by scanning tunneling microscopy and associated multiple tip effects. Appl. Phys. Lett.,52, 362-4; (c) Mizes, H.A. and Foster, J.S. (1989). Long-range electronic perturbations caused by defects using scanning tunneling microscopy. Science, 244, 559-62.

18. Coleman, R.V., Dai, Z., McNairy, W.W., et al. (1993). Methods of experimental physics. In Scanning Tunneling Microscopy. Vol. 27 (J.A. Stroscio and W.J. Kaiser, eds). Academic Press.

19. Mizes, H.A., Park, S., and Harrison, W.A. (1987). Multiple-tip interpretation of anomalous scanning-tunneling-microscopy images of layered materials. Phys. Rev. $B, 36,4491-4$.

20. Binnig, G., Fuchs, H., Gerber, Ch., et al. (1986). Energy-dependent state-density corrugation of a graphite surface as seen by scanning tunneling microscopy. Europhys. Lett., 1, 31-6.

21. Lin, F. and Meier, D.J. (1994). Atomic-scale resolution in atomic force microscopy. Langmuir, 10, 1660-2.

22. McDermott, M.T. and McCreery, R.L. (1994). Scanning tunneling microscopy of ordered graphite and glassy carbon surfaces: electronic control of quinone adsorption. Langmuir, 10, 4307-14.

23. Somorjai, A.G. (1981). Chemistry in Two Dimensions: Surfaces. Ithaca: Cornell University Press, p. 178.

24. Findengg, G.H. (1972). Ordered layers of aliphatic alcohols and carboxylic acids at the pure liquid/graphite interface. J. Chem. Soc. Faraday Trans., 68, 1799-806; Ikai, A. (1996). STM and AFM of bio/organic molecules and structures. Surf. Science Rep., 26, 261-332; Giancarlo, L.C. and Flynn, G.W. (1998). Scanning tunneling and atomic force microscopy probes of self-assembled, physisorbed monolayers: peeking at the peaks. Annu. Rev. Phys. Chem., 49, 297-336; Xie, Z.X., Xu, X., Mao, B.W., and Tanaka, K. (2002). Self-assembled binary monolayers of $n$-alkanes on reconstructed $\mathrm{Au}(111)$ and HOPG surfaces. Langmuir, 18, 3113-16.

25. (a) Zubimendi, J.L., Salvarezza, R.C, Vázquez, L., and Arvia, A.J. (1996). Scanning tunneling microscopy observation of sulfur electrodeposits on graphite single crystals. Langmuir, 12, 2-11; (b) Vicente, J.L. Mola, E.E., Appignanessi, G., et al. (1996). A quantum chemistry approach to possible sulfur adsorbate structures on the basal plane of graphite clusters. Langmuir, 12, 19-22.

26. Vericat, C., Andreasen, G., Vela, M.E., and Salvarezza, R.C. (2000). Dynamics of potential-dependent transformations in sulfur adlayers on $\mathrm{Au}(111)$ electrodes. J. Phys. Chem. B, 104, 302-7; Andreasen, G., Vericat, C., Vela, M.E., and Salvarezza, R.C. (1999). Dynamics of sulfur adlayer transformations at metal/electrolyte interfaces. J. Chem. Phys., 111, 9457-60.

27. Venkataraman, B., Flynn, G.W., Wilbur, J.L., et al. (1995). Differentiating functional groups with the scanning tunneling microscope. J. Phys. Chem., 99, 8684-9.

28. Cyr, D.M., Venkataraman, B., Flynn, G.W., et al. (1996). Functional group identification in scanning tunneling microscopy of molecular adsorbates. J. Phys. Chem., 100, 13747-59. 
29. Terán Arce, F., Vela, M.E., Salvarezza, R.C., and Arvia, A.J. (1996). Comparative study of thiol films on $\mathrm{C}(0001)$ and $\mathrm{Au}(111)$ surfaces by scanning probe microscopy. Surf. Rev. Lett., 4, 637-49.

30. Porter, M.D., Bright, T.B., Allara, D.L., and Chidsey, C.E.D. (1987). Spontaneously organized molecular assemblies. 4. Structural characterization of $n$-alkyl thiol monolayers on gold by optical ellipsometry, infrared spectroscopy, and electrochemistry. J. Am. Chem. Soc., 109, 3559-68.

31. Stranick, S.J., Parikh, A.N., Allara, D.L., and Weiss, P.S. (1994). A new mechanism for surface diffusion: motion of a substrate-adsorbate complex. J. Phys. Chem., 98, 11136-42.

32. Ulman, A. (1991). An Introduction to Ultrathin Organic Films: from Langmuir-Blodgett to Self-Assembly. Academic Press; Finklea H.O. (2000). Encyclopedia of Analytical Chemistry: Applications, Theory and Instrumentation (R.A. Meyers, ed.). Wiley; Schreiber, F. (2000). Structure and growth of self-assembling monolayers. Prog. Surf. Sci., 65, 151-257. 